Hysteresis loops and multi-stability: From periodic orbits to chaotic dynamics (and back) in diatomic granular crystals

This article has been downloaded from IOPscience. Please scroll down to see the full text article.

2013 EPL 10144003

(http://iopscience.iop.org/0295-5075/101/4/44003)

View the table of contents for this issue, or go to the journal homepage for more

Download details:

IP Address: 131.215.71.79

The article was downloaded on 04/04/2013 at 15:39

Please note that terms and conditions apply. 


\title{
Hysteresis loops and multi-stability: From periodic orbits to chaotic dynamics (and back) in diatomic granular crystals
}

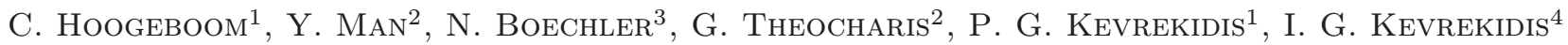 \\ and C. DARAIO ${ }^{2}$ \\ ${ }^{1}$ Department of Mathematics and Statistics, University of Massachusetts - Amherst, MA 01003-4515, USA \\ ${ }^{2}$ Graduate Aerospace Laboratories (GALCIT) California Institute of Technology - Pasadena, CA 91125, USA \\ ${ }^{3}$ Department of Mechanical Engineering, Massachusetts Institute of Technology - Cambridge, MA 02139, USA \\ ${ }^{4}$ Department of Chemical and Biological Engineering and PACM, Princeton University \\ Princeton, NJ, 08544, USA
}

received 2 November 2012; accepted in final form 20 January 2013

published online 28 February 2013

PACS 45.70.-n - Granular systems

PACS 63.20. Pw - Localized modes

PACS 05.45.-a - Nonlinear dynamics and chaos

\begin{abstract}
We consider a statically compressed diatomic granular crystal, consisting of alternating aluminum and steel spheres. The combination of dissipation, driving of the boundary, and intrinsic nonlinearity leads to complex dynamics. Through both numerical simulations and experiments, we find that the interplay of nonlinear surface modes with modes caused by the driver create the possibility, as the driving amplitude is increased, of limit cycle saddle-node bifurcations beyond which the dynamics of the system becomes chaotic. In this chaotic state, part of the applied energy can propagate through the chain. We also find that the chaotic branch depends weakly on the driving frequency, and speculate a connection between the chaotic dynamics with the gap openings between the spheres. Finally, we observe hysteretic dynamics and an interval of multi-stability involving stable periodic solutions and chaotic ones.
\end{abstract}

Copyright (c) EPLA, 2013

Introduction. - Granular systems, consisting of densely packed particles that interact through nonlinear, tensionless potentials, have been recognized in the last decade as a fertile testbed where ideas from nonlinear dynamics can be put to use [1,2]. Relevant investigations have focused on the dynamics of nonlinear waveforms, including traveling waves [1-8] and discrete breathers [9], as well as other nonlinear processes, including second harmonic generation and nonlinear resonances [10]. Several potential applications have been suggested, including energy absorbing layers [11-13], sound scramblers [14], acoustic lenses [15], and rectifiers [16].

In the present work, we explore the ability of granular crystals to support chaotic dynamics, which are accompanied by energy transmission, when subjected to external driving that is above a critical amplitude and has a frequency in the forbidden gap of its linear spectrum. Dissipation is abundant in the system and has been the subject of recent investigations [17-19]. By driving the system, we produce a case example of a dampeddriven system of coupled nonlinear oscillators. This system has the potential for the future study of pattern formation [20,21] as well as nonlinear supratransmission $[22,23]$, which can be triggered by the interplay of ordered (periodic, quasiperiodic) and chaotic dynamics [24].

Specifically, we study a one-dimensional, statically compressed diatomic system, consisting of alternating aluminum and steel spheres, where the first sphere is aluminum. In the case where the crystal starts with a steel sphere, its undamped and undriven Hamiltonian analogue does not support nonlinear surface modes, which leads to a drastic modification of the bifurcation features and dynamics detailed below. In our case, the presence of driving at the boundary and its interaction with dissipation, nonlinearity, and discreteness, enables two classes of relevant states: nonlinear surface modes and states tuned to the external driver. These two branches of time-periodic solutions are observed to collide and disappear in a limit cycle saddle-node bifurcation. Beyond 


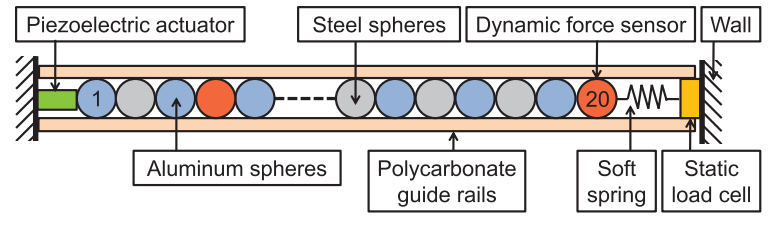

Fig. 1: (Color online) Schematic of the experimental setup.

this critical point, no stable, periodic solutions are found to exist, and the dynamics "jump" to a chaotic branch as the strength of the drive is further increased. This jump is accompanied by the onset of energy transmission through the chain. As the drive is then decreased, a hysteretic loop is found to arise, with the chaotic solutions persisting well below the saddle-node point. Such a hysteretic loop between periodic solutions and chaotic ones can be found in other physical systems ranging from nonlinear optics (e.g., dissipative soliton molecules in mode-locked fiber lasers [25]) to droplet dynamics (e.g., in dripping faucets $[26])$.

Experimental setup. - We construct a diatomic granular crystal, as shown in fig. 1 , by alternating $N=20$ aluminum spheres (6061-T6 type, radius $R_{a}=9.53 \mathrm{~mm}$, mass $m_{a}=9.75 \mathrm{~g}$, elastic modulus $E_{a}=73.5 \mathrm{GPa}$, Poisson ratio $\left.\nu_{a}=0.33\right)$ and stainless-steel spheres (316 type, $\left.R_{b}=R_{a}, m_{b}=28.84 \mathrm{~g}, E_{b}=193 \mathrm{GPa}, \nu_{b}=0.3\right)[27,28]$. We constrain the spheres to a one-dimensional configuration by using four polycarbonate rods arranged in a square pattern, which are aligned by periodically spaced guide plates. On a steel block fixed to the table at one end of the granular crystal, we mount a piezoelectric actuator that applies dynamic displacements to the crystal boundary. We calibrate the displacement of the actuator tip as a function of driving voltage by using a laser vibrometer in conjunction with a strain-gauge embedded in the actuator. The particle next to the actuator is an aluminum particle. At the opposite end of the crystal from the actuator, we apply a static load $\left(F_{0}=8 \mathrm{~N}\right)$ by compressing a soft spring (stiffness $1.24 \mathrm{kN} / \mathrm{m}$ ) between the last particle of the crystal, and a static load cell mounted in a teflon holder. The spring, static load cell, and granular crystal are compressed by fixing a second steel block to the table at some distance relative to the other block. We measure the propagating stress waves with calibrated piezoelectric disks epoxied inside two halves of a steel sphere (maintaining properties such as inertia and bulk stiffness of the original steel spheres) [11]. For our frequencies of interest, each sensor measures the instantaneous average dynamic force between each of its two adjacent contacts. We place sensor particles at sites $n=4$ and $n=20$. The signals are filtered using a low-pass filter and amplifier $(30 \mathrm{kHz}$, 8th-order Butterworth low-pass filter) and recorded with a data acquisition board. The signals are then digitally filtered with a $100 \mathrm{~Hz}$ 5th-order Butterworth high-pass filter to remove electrical noise.
Model. - We model our damped and driven granular crystal as the following set of equations:

$$
\begin{gathered}
m_{j} \ddot{u}_{j}=A_{j-1, j}\left[\delta_{j-1, j}-\left(u_{j}-u_{j-1}\right)\right]_{+}^{3 / 2} \\
-A_{j, j+1}\left[\delta_{j, j+1}-\left(u_{j+1}-u_{j}\right)\right]_{+}^{3 / 2}-\frac{m_{j} \dot{u}_{j}}{\tau},
\end{gathered}
$$

where $m_{j}$ is the mass of the $j$-th bead, $A_{j, j+1}=$ $4 E_{j} E_{j+1} \sqrt{\frac{R_{j} R_{j+1}}{R_{j}+R_{j+1}}}$ $\frac{3 E_{j+1}\left(1-\nu_{j}^{2}\right)+3 E_{j}\left(1-\nu_{j+1}^{2}\right)}{R_{j}}$ is the Hertzian contact coefficient, and $\delta_{j, j+1}=\left(F_{0} / A_{j, j+1}\right)^{2 / 3}$ is the amount of static overlap (due to the precompression) between beads $j$ and $j+1[1]$. The actuator is placed at the left edge of the chain and provides a prescribed displacement with a frequency $f_{d}$ and amplitude $\alpha$ given by $u_{0}(t)=\alpha \cos \left(2 \pi f_{d} t\right)$. The right edge of the chain is stress-free. Brackets $[Y]_{+}$denote the positive part of $Y$, which signifies that adjacent particles interact only when they are in contact. We model linear on-site dissipation with time constant $\tau=1.75$ $\mathrm{ms}$, in line with ref. [16]. The linear spectrum of the undamped, undriven crystal has an acoustic band and an optical band, with a finite gap between, and a semi-infinite gap above the optical band [9]. For a precompressive force of $F_{0}=8 \mathrm{~N}$, and the cited material parameters, the acoustic band is predicted to run from $0 \mathrm{kHz}$ to $3.98 \mathrm{kHz}$, and the optical band from $6.96 \mathrm{kHz}$ to $8.02 \mathrm{kHz}$.

Numerical results and analysis. - Our main theoretical findings are summarized in the bifurcation diagram and stability analysis of fig. 2, which has been computed for $f_{d}=6 \mathrm{kHz}$. We find periodic solutions to the system, using the amplitude of the actuator as a bifurcation parameter (for fixed frequency) in our Newton algorithm for the system of eq. (1). Other examples of this method for granular crystals can be found in refs. [9,16]. The bifurcation diagram has three principal branches of periodic solutions, as shown in fig. 2(a). The branch represented by the solid (blue) line corresponds to modes that are absent when the actuator is off. The branches represented by the dashed (red) and dash-dotted (green) lines stem from a finite force, well above the precompression threshold, and emerge through a limit cycle saddle-node bifurcation at actuator amplitude $\alpha_{c}^{(0)} \approx 0.06 \mu \mathrm{m}$. Without dissipation these two branches merge at $\alpha=0$, as is shown in the left inset of fig. 2(a). Both branches emerge as a driven continuation of nonlinear modes, which are the surface analogues of intrinsic bulk breathers [9]. The finite critical threshold $\left(\alpha_{c}^{(0)}\right)$ for their existence is due to the inability of the undriven crystal to sustain large amplitude localized modes in the presence of finite damping. The stability analysis for these three branches is shown in fig. 2(b)-(d). Figure 2(b) corresponds to the dash-dotted (green) branch in fig. 2(a). This branch bears quartets of complex multipliers, with the exception of two very small intervals of actuator amplitudes (in a neighborhood of the initial bifurcation point and of driving amplitude $0.2 \mu \mathrm{m}$ ), and so it is oscillatorily unstable. Figure 2(c) corresponds to the dashed (red) branch, which always bears at least a 

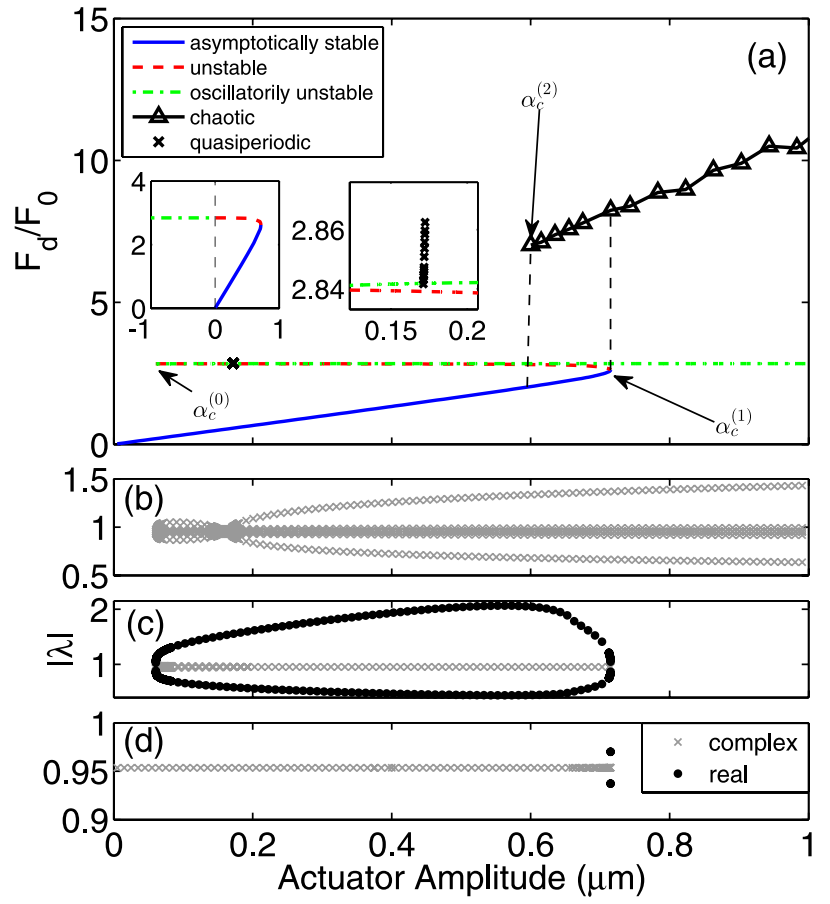

Fig. 2: (Color online) (a) Numerical bifurcation diagram of average dynamical force at the fourth site of the chain normalized against the static load $F_{0}$, continued as a function of actuator amplitude. The three time-periodic branches are shown by the lines without markers. Past $\alpha=\alpha_{c}^{(2)}$, an additional chaotic branch also exists, denoted by the line with triangle markers. A Hopf bifurcation that spawns a branch of quasiperiodic solutions is shown by an " $\times$ " marker. An enlarged view of the Hopf bifurcation is shown in the right inset. The left inset shows the corresponding bifurcation diagram for the undamped system, where the unstable branch reaches $\alpha=0 \mu \mathrm{m}$ and extends towards the osillatorily unstable branch for $\alpha<0$. The bottom three panels show the moduli of the Floquet multipliers for the oscillatorily unstable dash-dotted branch (b), the unstable dashed branch (c), and the stable solid branch $(d)$.

real pair of multipliers with $|\lambda|>1$, and so it is unstable. Figure 2(d) corresponds to the solid (blue) branch, which is asymptotically stable, as illustrated by the absence of real (or complex) Floquet multipliers.

Typical profiles of the nonlinear localized modes for each of the three periodic branches of fig. 2 , at time $t=0$ with $\alpha=0.4 \mu \mathrm{m}$, are shown in fig. 3. These nonlinear surface modes are similar to the profiles of hybrid bulksurface localized solutions (see fig. 9 in ref. [9]). The first particle of the modes corresponding to the solid (blue) and dashed (red) branches of fig. 2 is essentially in phase with the driver, while that of the dash-dotted (green) branch is out-of-phase with the actuator. The driver is cosinusoidal, and bears a positive amplitude at $t=0$. A feature of interest is that the low-amplitude branch due to the actuator, and the unstable dashed branch due to the system's intrinsic linear and nonlinear properties [9], which collide and disappear at amplitude $\alpha_{c}^{(1)} \approx 0.71 \mu \mathrm{m}$. Past

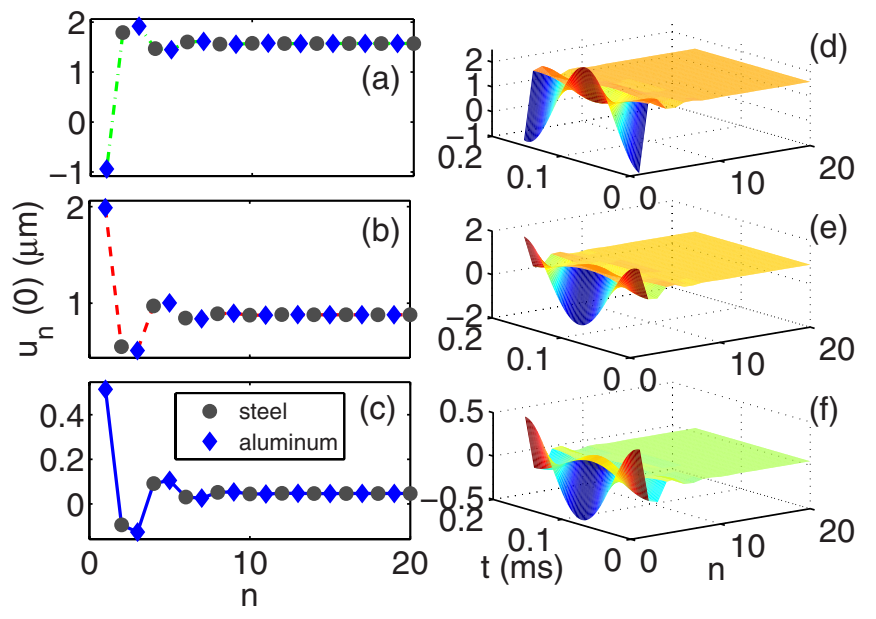

Fig. 3: (Color online) Sample profiles at $t=0$ (left panels) and spatiotemporal evolution of the solutions (right panels) for each of the three breather branches are shown for $\alpha=0.4 \mu \mathrm{m}$. Panels (a), (d) correspond to the oscillatorily unstable dashdotted branch; (b), (e) to the unstable dashed branch, and (c), (f) to the stable solid branch.

this critical point, we observe a chaotic large-amplitude response, which appears to exist for amplitudes $\alpha>\alpha_{c}^{(1)}$.

In order to identify the domain of existence of the chaotic branch, we continued this branch for $\alpha<\alpha_{c}^{(1)}$, and identified the existence of such solutions down to $\alpha_{c}^{(2)} \approx$ $0.59 \mu \mathrm{m}$. However, these solutions are obtained via numerical integration of the equations of motion only and are not numerically exact solutions. For instance, taking an initial condition close to the chaotic attractor, we then integrate it forward in time. If the solution stays close to the chaotic attractor after 4000 oscillations of the actuator, then we designate this solution part of the chaotic branch. For amplitudes $\alpha<\alpha_{c}^{(2)}$ even large-amplitude initial data was found to asymptotically approach the low-amplitude periodic solution of the system (prior to the 4000 oscillation cutoff). This point thus signals the boundary of existence (or at least of dynamical stability/attractivity within this reporting horizon) of the relevant chaotic branch. Between these two critical points, i.e., for amplitudes $\alpha_{c}^{(2)}<\alpha<\alpha_{c}^{(1)}$, the dynamics of the system is multistable. For low initial amplitudes, the dynamics converges to the small amplitude periodic solutions, while for large amplitudes, it is attracted to the chaotic orbits shown in fig. 2. For amplitudes $\alpha>\alpha_{c}^{(2)}$ and suitable initial data, positive Lyapunov exponents have been found to corroborate the chaotic nature of the relevant orbits. The separatrix between low-amplitude--ordered dynamics and largeamplitude chaotic ones appears to be associated with the oscillatorily unstable dash-dotted (green) breather branch, depicted in fig. 2. It is conceivable that the stable and (low-dimensional) unstable manifolds of this branch are involved in the "demise" of the chaotic attractor observed in our simulations. 
Experimental results. - We experimentally characterize the near-linear response of our granular crystal, as described in ref. [9], by measuring the linear spectrum of the crystal by applying low-amplitude (approximately $5 \mathrm{mN}$ peak), broadband frequency $(2-18 \mathrm{kHz})$, uniform noise for $1040 \mathrm{~ms}$ with the piezoelectric actuator. Using the force signal measured at each sensor location, we then calculate the power spectral density (PSD) of the central portion of the force response (524 ms duration), and normalize by the PSD of the voltage signal applied to the actuator, and average this over 8 trials. The cutoff frequencies are then determined by finding frequencies where the attenuation is greater than $-10 \mathrm{~dB}$ from the mean level between $2-4 \mathrm{kHz}$ (which corresponds to the center of the acoustic band). The spectra was measured before and after the bifurcation experiments in order to characterize any change in the cutoff frequencies due to the dynamic loading of the crystal. The spectra measured before single frequency experiments exhibited an acoustic band cutoff frequency of $4.37 \mathrm{kHz}$, a lower optical band cutoff frequency of $7.83 \mathrm{kHz}$, and an upper optical band cutoff frequency of $8.50 \mathrm{kHz}$. The cutoff frequencies show a systematic upshift of about $9 \%$ compared to the theoretical predictions. Possible explanations can be found in ref. [9], where a similar upshift has been observed. A further upshift was also observed after driving the system at high amplitude $(1.2 \mu \mathrm{m}$ driving amplitude at $6 \mathrm{kHz})$. This gave cutoff frequencies of $4.83,8.54$, and $9.45 \mathrm{kHz}$, respectively. We speculate this could be caused by slight dynamic rearrangement of the granular crystal under high loading conditions. Under such conditions, particles are expected to lose contact, and the crystal may reassemble into an arrangement with slightly modified off-axis displacements.

Following measurement of the linear spectrum, we experimentally characterize the bifurcation of the system response by exciting the crystal with a single frequency signal $(6,6.5$, and $6.8 \mathrm{kHz})$. Each signal is $90 \mathrm{~ms}$ in duration (where the amplitude of the signal is linearly increased and decreased during the first and last $20 \mathrm{~ms}$, respectively). For a given driving frequency, we sequentially increase the maximum amplitude of the driving signal, and record the dynamic force, $F_{i}$, measured by the sensor at particle $n=4$. Examples of the measured force-time response are shown in fig. 4(a) and (b). In fig. 5(a) the average dynamic force $F_{d}$ is $\sqrt{2}$ times the root mean square (RMS) variation of the time interval highlighted in blue (from $t=90 \mathrm{~ms}$ to $t=122.8 \mathrm{~ms}$ ) of the force-time history. In fig. $5(\mathrm{a})$, we plot the dynamic force amplitude (normalized against the static load) as a function of actuator displacement, for driving frequencies of $f_{d}=6,6.5$, and $6.8 \mathrm{kHz}$. For each driving frequency $(6 \mathrm{kHz}$ (black triangles), $6.5 \mathrm{kHz}$ (red crosses), and $6.8 \mathrm{kHz}$ (blue circles)), after the driving amplitude crosses a certain threshold, the system jumps from a low-amplitude stable periodic response to a high-amplitude chaotic response (see also fig. 4(a) and (b)). This transition is also

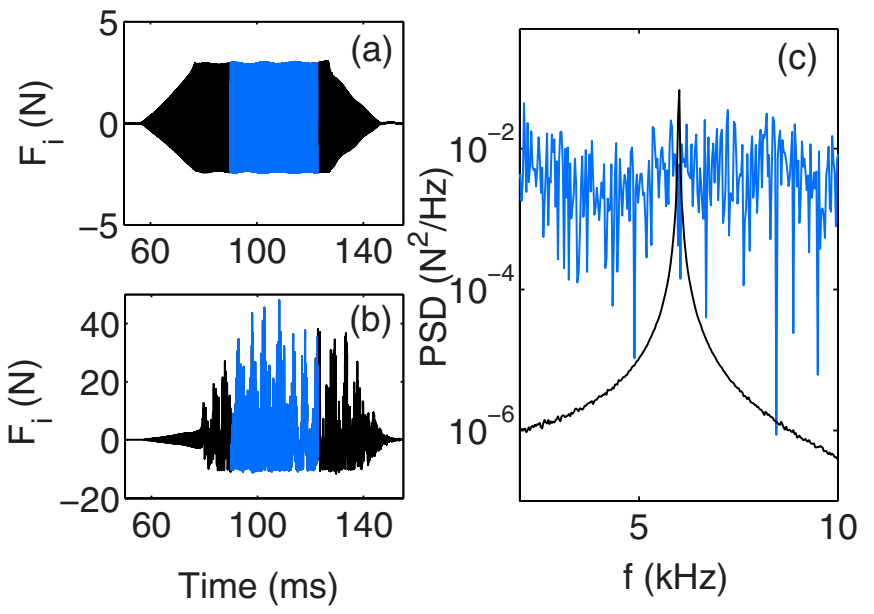

Fig. 4: (Color online) Experimental force-time histories measured by the sensor at $n=4$, corresponding to (a) the largest low-amplitude periodic response (blue square in fig. 5), and (b) the first amplitude following the onset of chaos (blue diamond in fig. 5). The central blue region highlights the time interval over which the PSD was calculated. (c) The black and blue curves are the PSD of the signals in (a) and (b), respectively.

characterized by a drastic change in the frequency content of the response, as shown in fig. 4(c), from that of a single frequency response, before the critical point, that corresponds to the driving frequency (black), to a broadband frequency response (blue) afterwards. The change in the frequency content of the response implies partial transfer of the applied energy to frequencies in the propagating bands. A similar phenomenon, although of a different origin, was observed in chains of coupled pendula [22].

This behavior is similar to that of the numerical results shown in the right panel of fig. 5, where an increase in driving frequency corresponds to a decrease in the critical threshold $\left(\alpha_{c}^{(1)}\right)$. Quantitative differences between experiments and numerics can be explained by the differences between theoretical and experimental spectra. Driving the crystal at $6 \mathrm{kHz}$ numerically, where the lower cutoff frequency is at $6.96 \mathrm{kHz}$, is not equivalent to driving it at $6 \mathrm{kHz}$ experimentally, when the cutoff frequencies in experiments are higher. Other simplifications in the modeling of the setup, such as the approximation of dissipation with a linear on-site dissipative term and the assumption of a purely one-dimensional crystal geometry may also contribute.

Figure 5(c) also shows that the chaotic branch is similar for both frequencies. In our simulations, we observed this to be generic. The chaotic branch appears similar for large actuator amplitudes, independent of the driving frequency. We also observed that the higher the driving frequency, the larger the domain of existence of the chaotic branch $\left(\alpha_{c}^{(2)}\right.$ is smaller). Figure 5(a) shows a similar trend in the experiments. Because of this, we suggest the chaotic 

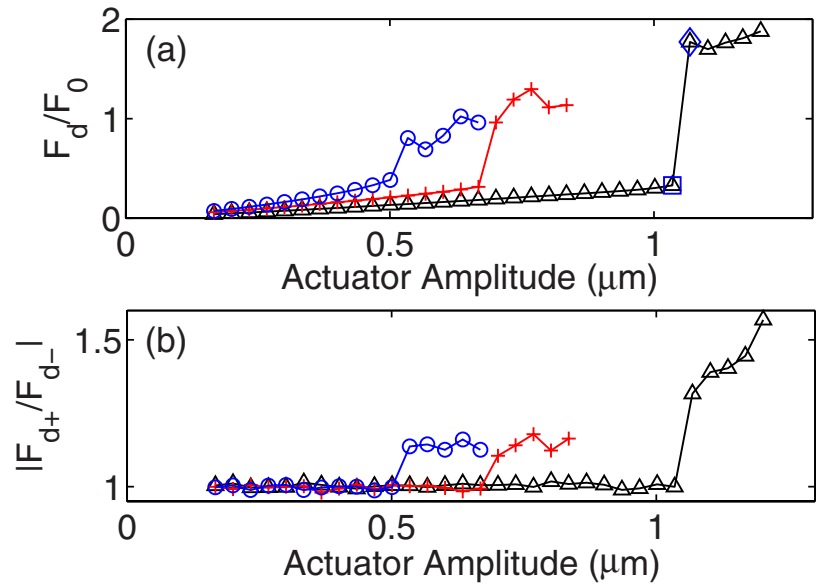

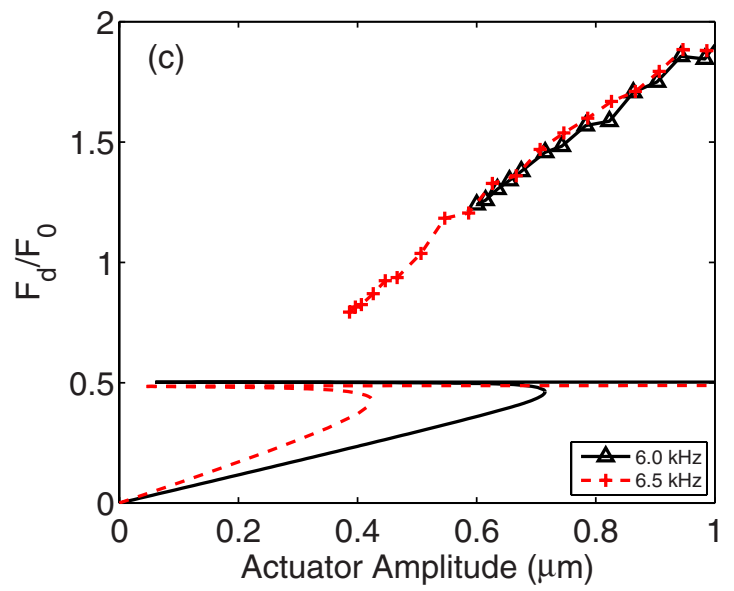

Fig. 5: (Color online) (a) Dynamic force amplitude at driving frequencies of $6 \mathrm{kHz}$ (black triangles), $6.5 \mathrm{kHz}$ (red crosses), and $6.8 \mathrm{kHz}$ (blue circles). The blue square corresponds to the force-time history immediately before the onset of chaos (fig. 4(a)), while the blue diamond corresponds to the force-time history immediately following the onset of chaos (fig. 4(b)). (b) Asymmetry of the force-time response, as the actuator displacement is increased. The onset of asymmetry (as $\left|F_{d+} / F_{d-}\right|$ becomes $>1$ ) corresponds to the onset of instability for all three driving frequencies. (c) Numerically obtained dynamic force amplitude at driving frequencies of $6 \mathrm{kHz}$ (black solid line with triangles) and $6.5 \mathrm{kHz}$ (red dashed line with crosses). In each case, lines without markers are numerically exact periodic solutions, while the lines with markers are the chaotic branches.
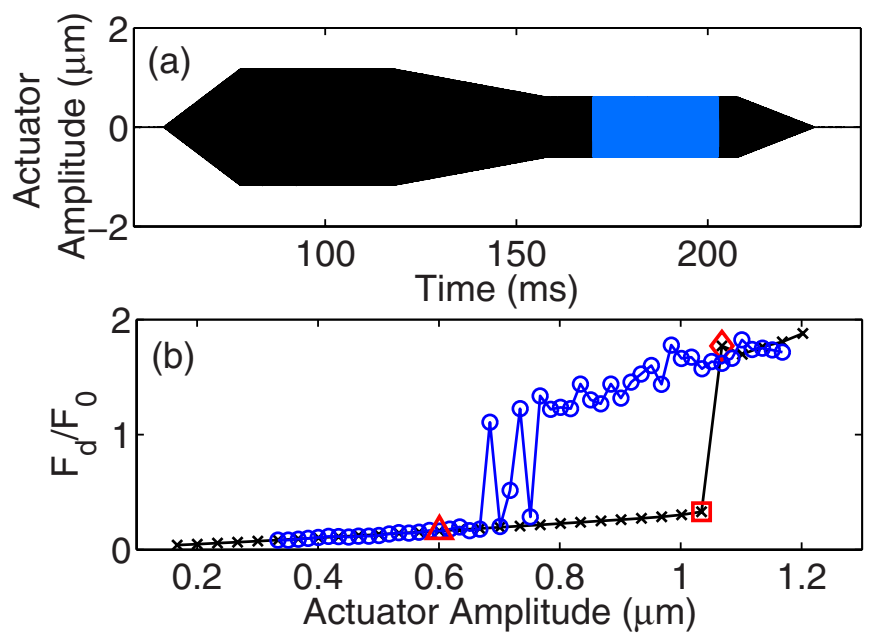

Fig. 6: (Color online) (a) The hysteresis loop driving amplitude for the run marked by the red triangle in (b). The initial driving amplitude is $1.2 \mu \mathrm{m}$ for all experiments. The blue region highlights the time interval over which the PSD was calculated. (b) Experimentally characterized hysteresis loop showing dynamic force as a function of actuator driving amplitude, at a driving frequency of $6 \mathrm{kHz}$. Black " $\times$ " markers denote following the stable periodic solution to chaos, while blue circles denote the return from a chaotic response back to the low-amplitude periodic response. The red square and diamond markers correspond to the blue square and diamond, respectively, of fig. $5(\mathrm{a})$.

branch is less sensitive to the driving frequency and depends mostly on gap openings between the beads. This is supported by the observed asymmetry of the forcetime response, shown in fig. 5(b). The asymmetry of the force-time response is indicative of dynamic loss of contact of particles in the granular crystal. Using the same portion of the force signal used to calculate the PSD, we separated the dynamic force signal into its positive and negative values, calculated the RMS of both $\left(F_{d+}\right.$ and $F_{d-}$, respectively), and found the absolute value of the ratio between them, to give an effective measure of asymmetry. When the forces are symmetric, we expect this ratio to be close to 1 . However, after instability occurs and the system is driven to its chaotic branch of solutions, the ratio jumps to a substantially larger value. This phenomenon is seen in all three driving frequencies, and the onset of asymmetry occurs at the same actuator displacements as the systems' transition to chaos.

After measuring the bifurcation of the system response for increasing amplitudes and $6 \mathrm{kHz}$ driving, we characterize the return part of the hysteresis loop, as shown in fig. 6. We drive the system at $6 \mathrm{kHz}$ for a duration of $170 \mathrm{~ms}$. The envelope of the signal is linearly ramped up for $20 \mathrm{~ms}$ to a voltage high enough to induce chaos. This amplitude is held constant for $40 \mathrm{~ms}$, and then is linearly ramped down (over $40 \mathrm{~ms}$ ) to a lower secondary amplitude (which is gradually decreased over each sequential run) that continues for $50 \mathrm{~ms}$. Finally, the last $20 \mathrm{~ms}$ are linearly ramped down to zero amplitude. These ramps allow us to track the low-amplitude stable periodic branch until its disappearance, then follow the chaotic solution down to lower amplitudes, until the system response drops back to the low-amplitude branch of periodic solutions. The average dynamic force is calculated and measured as described previously, except that the middle of the secondary constant amplitude region is used (from $t=170 \mathrm{~ms}$ to $t=202.8 \mathrm{~ms}$, as shown in the top panel of fig. 6). The experimentally measured hysteresis loop is shown in fig. 6(b). As we track the chaotic branch downwards, there is an intermediate region over which the solutions are observed to alternate between chaotic and 
periodic responses (from approximately $0.7 \mu \mathrm{m}$ to $0.8 \mu \mathrm{m}$ ), before settling back to the stable low-amplitude periodic branch. This may be a sign of a transient chaotic regime. For longer driving at the secondary amplitude longer, the system may eventually fall back to the periodic response. This could also be attributed to small differences in the geometry of the chain from measurement to measurement induced by rearrangement of the granular crystal due to the high-amplitude dynamic loading.

Conclusions and future challenges. - In this work, we provided a prototypical example of the interplay of the damped-driven dynamics (at least partially extrinsic to the chain) and the periodic nonlinear dynamics (chiefly intrinsic to the chain) that give rise to saddle-node bifurcations of periodic solutions and turning points beyond which there is no stable ordered dynamics. These transitions can be controlled by parameters such as the frequency and the amplitude of the drive. Past these points, the response of the system is chaotic, yet it can also be chaotic well before that, creating regimes of multi-stability and associated separatrices between time-periodic and genuinely chaotic attractors. This study provides a gateway for the exploration and utilization of the capability of the system to exhibit large-amplitude chaotic dynamics, and (even concurrently) periodic dynamics.

Nevertheless, numerous interesting questions remain unexplored. The mechanism leading to the emergence of chaotic dynamics warrants further investigation. An avenue that we have not explored in detail herein (but will present in detail in a future investigation) involves the emergence of quasiperiodic solutions from the critical (Hopf) point of actuator amplitude $\alpha \approx 0.17 \mu \mathrm{m}$. It is plausible - yet presently unclear - that sequences of global bifurcations destroy these tori, and eventually lead to chaotic dynamics. It is also conceivable that the stable and unstable manifolds of the oscillatorily unstable (upper) branch of periodic solutions are involved in the emergence, as the drive amplitude is increased (and disappearance as it is decreased), of the apparent chaotic attractors. This intriguing, yet technically challenging, theme will be deferred to future investigations.

Support from US-NSF, US-AFOSR and the A.S. Onassis Foundation is appreciated.

\section{REFERENCES}

[1] Nesterenko V. F., Dynamics of Heterogeneous Materials (Springer-Verlag, New York) 2001.

[2] Sen S. et al., Phys. Rep., 462 (2008) 21.

[3] Nesterenko V. F., Prikl. Mekh. Tekh. Fiz., 24 (1983) 136 (J. Appl. Mech. Tech. Phys., 24 (1984) 733).

[4] Coste C., Falcon E. and Fauve S., Phys. Rev. E., 56 (1997) 6104.

[5] Porter M. A. et al., Phys. Rev. E, 77 (2008) 015601(R).

[6] Starosvetsky Y. and Vakakis A. F., Phys. Rev. E, 82 (2010) 026603.

[7] Jayaprakash K. R., Starosvetsky Y. and Vakakis A. F., Phys. Rev. E, 83 (2011) 036606.

[8] JAMES G., J. Nonlinear Sci., 22 (2012) 813, DOI: 10.1007/s00332-012-9128-3; see also arXiv:1203.0761.

[9] Boechler N. et al., Phys. Rev. Lett., 104 (2010) 244302; Phys. Rev. E, 82 (2010) 056604.

[10] Cabaret J., Tournat V. and Bequin P., Phys. Rev. E, 86 (2012) 041305.

[11] Daraio C. et al., Phys. Rev. Lett., 96 (2006) 058002.

[12] Hong J., Phys. Rev. Lett., 94 (2005) 108001.

[13] Doney R. and Sen S., Phys. Rev. Lett., 97 (2006) 155502.

[14] Nesterenko V. F. et al., Phys. Rev. Lett., 95 (2005) 158702.

[15] Spadoni A. and Daraio C., Proc. Natl. Acad. Sci. U.S.A., 107 (2010) 7230.

[16] Boechler N., Theocharis G. and Daraio C., Nat. Mater., 10 (2011) 665.

[17] Rosas A. et al., Phys. Rev. Lett., 98 (2007) 164301.

[18] Carretero-González R. et al., Phys. Rev. Lett., 102 (2009) 024102.

[19] Vergara L., Phys. Rev. Lett., 104 (2010) 118001.

[20] Khomeriki R., Lepri S. and Ruffo S., Phys. Rev. E, 64 (2001) 056606.

[21] Cross M. C. and Hohenberg P. C., Rev. Mod. Phys., 65 (1993) 851.

[22] Geniet F. and Leon J., Phys. Rev. Lett., 89 (2002) 134102

[23] Khomeriki R., Lepri S. and Ruffo S., Phys. Rev. E, 70 (2004) 066626.

[24] Maniadis P., Kopidakis G. and Aubry S., Physica D, 216 (2006) 121.

[25] Zavyalov A. et al., Opt. Lett., 34 (2009) 3827.

[26] Kiyono K. and Fuchikami N., J. Phys. Soc. Jpn., 71 (2002) 49.

[27] Boechler N. et al., J. Appl. Phys., 109 (2010) 074906.

[28] Man Y. et al., Phys. Rev. E, 85 (2012) 037601. 